\title{
Prospective evaluation of FISH for detecting upper tract urothelial carcinoma in voided urine specimens
}

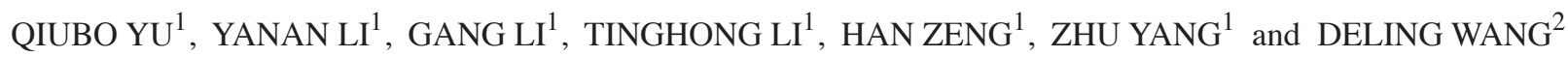 \\ ${ }^{1}$ Cell Genetics Laboratory, Molecular Medical Laboratory Center; ${ }^{2}$ Department of Urological Surgery, \\ The First Affiliated Hospital, Chongqing Medical University, Chongqing 400016, P.R. China
}

Received March 16, 2015; Accepted March 22, 2016

DOI: $10.3892 / \mathrm{ol} .2016 .4592$

\begin{abstract}
The aim of the present study was to estimate the diagnostic value of fluorescence in situ hybridization (FISH) analysis of tumor cells in voided urine specimens for detecting upper tract urothelial carcinoma (UTUC). Cytology and FISH analyses were conducted on voided urine collected in the morning from 125 patients with suspected UTUC. During follow-up, ureteroscopy with biopsy and histopathology were used to confirm the presence of tumors. The average follow-up time was 23.8 months (range, 6-36 months). A total of 8 patients who could not be contacted until the last follow-up were excluded from the study. Of the remaining 117 patients, 19 were histologically demonstrated to have UTUC, of whom, 3 patients had stage pTis disease, 6 had stage pTa disease, 5 had stage pT1 disease and 5 had stage pT2 disease (7 G1, 8 G2 and 4 G3). The overall sensitivity of FISH to detect UTUCs in voided urine specimens was $84.21 \%$ (16/19), whereas that of cytology was $42.11 \%(8 / 19)(\mathrm{P}<0.05)$. The overall specificity of FISH to detect UTUCs in voided urine specimens was $89.80 \%$ (88/98), compared with $94.90 \%$ (93/98) of cytology $(\mathrm{P}>0.05)$. The positive predictive value (PPV) and negative predictive value of FISH were $80.00 \%(16 / 20)$ and $97.78 \%$ (88/90), respectively, whereas those of cytology were $100.00 \%$ $(8 / 8)(\mathrm{P}>0.05)$ and $90.29 \%(93 / 103)(\mathrm{P}>0.05)$, respectively. The present data indicated that FISH was a method capable of detecting UTUCs in voided urine specimens with good sensitivity and specificity, although it exhibited a high rate of false positivity and low PPV.
\end{abstract}

Correspondence to: Dr Qiubo Yu, Cell Genetics Laboratory, Molecular Medical Laboratory Center, Chongqing Medical University, 1 Yixueyuan Road, Yuzhong, Chongqing 400016, P.R. China

E-mail: yqb76712@gmail.com

Professor Deling Wang, Department of Urological Surgery, The First Affiliated Hospital, Chongqing Medical University, 1 Yixueyuan Road, Yuzhong, Chongqing 400016, P.R. China

E-mail: dlwangws@sina.com

Key words: fluorescence in situ hybridization, upper tract urothelial carcinoma, prospective, voided urine specimen

\section{Introduction}

Cancerous lesions of the renal pelvis and ureter are malignant tumors that arise from the urothelial (transitional cell epithelium) mucosa of the renal pelvis or ureter (1). Primary urinary tract urothelial carcinomas (UTUCs) are rare tumors with an estimated incidence of 1-4 cases/100,000 individuals/year (1). UTUC is much less common than bladder cancer, and only accounts for $\sim 5 \%$ of all urothelial carcinoma cases (2). A total of $90 \%$ of UTUCs are transitional cell carcinomas (2). Ureteral tumors are particularly rare $(1,3)$, and their incidence rate is $\sim 1 / 4$ of that of renal pelvic tumors (1).

The majority of patients with UTUC present with flank pain or hematuria at the early stages of the disease (4). Suspected UTUCs are usually evaluated with intravenous pyelography, retrograde pyelography (RGP), ultrasonography (UG), computed tomography (CT), upper tract urinary cytology and cystourethroscopy with biopsy $(2,4)$. Imaging studies usually reveal a filling defect or an obstructive mass, which is often associated with hydronephrosis, hydroureter or renal stones (2). Therefore, despite being non-invasive methods, imaging studies should not be used as the sole diagnostic tool for UTUC, due to their low sensitivity for the detection of small tumors, since numerous causes exist to explain a filling defect other than UTUC $(4,5)$. Cytology has a low sensitivity for detecting UTUC, and ureteroscopy is an invasive method with poor sensitivity, particularly in flat tumors $(4,6)$. The epithelium that covers the renal pelvis, bladder and ureter is mainly transitional. Various tumors may appear in the kidney area, particularly in the renal medulla portion of the kidney, and often require to be identified as potential transitional cell carcinoma of the renal pelvis (4). In addition, renal pelvis cancer and blood clots in the renal pelvis are often difficult to identify by imaging techniques (5).

Due to the above mentioned shortcomings, the identification of sensitive molecular markers for the detection of UTUC is urgently required. To date, only a limited number of sensitively accepted markers have been applied to detect urinary tumors, including ImmunnoCyt ${ }^{\mathrm{TM}} / \mathrm{uCyt}+{ }^{\mathrm{TM}}$ and fluorecence in situ hybridization (FISH) technology (7-9). FISH technology is generally used for the diagnosis of bladder cancer in voided urine in China, due to its high sensitivity and specificity, but its use for detecting UTUCs is limited, due to its low reliability (10). Luo et al (10) reviewed the utility of 
FISH in the diagnosis of UTUC, but this type of prospective study has not been conducted in China thus far.

Thus, in the present study, the sensitivity and specificity of FISH technology for detecting suspicious UTUCs in voided urine specimens was prospectively estimated, since this type of specimens are easily collected and accepted by patients.

\section{Materials and methods}

Patients and samples. Between May 2011 and August 2014, voided urine from 125 patients (82 males and 43 females; mean age, 61.4 years; range, 45-92 years) with suspicion of UTUC were analyzed prospectively. Patients were recruited from The First, The Second and The Fifth Affiliated Hospitals of Chongqing Medical University (Chongqing, China). Patients with clinical symptoms (gross hematuria, flank pain) and/or radiographic (UG or CT) abnormalities suggestive of UTUC, localising hematuria, such as blood efflux from the ureteral orifice, and atypical obstruction were included in the study. The patients were followed up for a mean \pm standard deviation observation time of 23.8 \pm 7.5 months (range, 5-39 months). First-time voided urine specimens, which were the first morning urine specimens at the time of admission to hospital, were collected for FISH and cytology tests in the morning. Urinary cells from voided urine were sedimented at $600 \mathrm{~g}$ for 10 min in a Sorvall Legend Mach 1.6R benchtop centrifuge (Thermo Fisher Scientific, Inc., Waltham, MA, USA), and the cell pellets were divided into two equal volumes, one of which was used for FISH and one for cytology analyses. The ethics committee of Chongqing Medical University (Chongqing, China) approved the present study, and all patients provided their informed consent prior to participation.

Cytology. Urinary cytology was performed according to the Papanicolau method (11), using an eosin alcohol 50 solution (Hubei Taikang Medical Equipment Co., Ltd., Hubei, China). Cytology was scored as positive, negative or suspicious by a senior cytologist. Suspicious cytology was defined as those samples that contained cells with morphologies that could not be clearly classified as tumor or normal cells. Histopathological classification was performed according to the Union for International Cancer Control criteria $(8,9)$.

FISH. Cell pellets were resuspended in $10 \mathrm{ml}$ preheated hypotonic solution $[0.075 \mathrm{~mol} / 1$ potassium chloride (Hubei Taikang Medical Equipment Co., Ltd.)], and incubated at $37^{\circ} \mathrm{C}$ for $25 \mathrm{~min}$, pipetting three times during this period of time to ensure a sufficient hypotonic environment for the cells. Next, cells were sedimented at $600 \mathrm{~g}$ for $10 \mathrm{~min}$, and the cell pellets were fixed in 3:1 (v/v) methanol: glacial acetic acid (Hubei Taikang Medical Equipment Co., Ltd.). The final cell pellet was resuspended in the appropriate volume of fixing liquid, according to the number of urinary cells. One or two drops of the resuspended cells $\left(10^{4}-10^{6}\right.$ cells $\left./ \mathrm{ml}\right)$ were seeded onto two glass slides (Hubei Taikang Medical Equipment Co., Ltd.) and incubated at $60^{\circ} \mathrm{C}$ for $2 \mathrm{~h}$.

The glass slides were then incubated at $37^{\circ} \mathrm{C}$ with RNase A solution [40 ml 2X saline-sodium citrate (SSC) solution with $60 \mu 1100 \mathrm{~g} / \mathrm{ml}$ RNase A; Beijing GP Medical Technologies, Ltd. (Beijing, China)] for $30 \mathrm{~min}$, followed by incubation with pepsin solution [40 ml 0.01 M HCl (Hubei Taikang Medical Equipment Co., Ltd.) with $160 \mu 120 \mathrm{mg} / \mathrm{ml}$ pepsin (Beijing GP Medical Technologies, Ltd.)] at $37^{\circ} \mathrm{C}$ for $10 \mathrm{~min}$. The slides were then washed twice in $2 \mathrm{X} \mathrm{SSC}$ solution for 5 min each, and sequentially dehydrated in 70,85 and $100 \%$ ethanol (3 min each) (Hubei Taikang Medical Equipment Co., Ltd.). The slides were then denatured in $70 \%$ formamide (Hubei Taikang Medical Equipment Co., Ltd.)/2X SSC denaturing solution at $73^{\circ} \mathrm{C}$ for $5 \mathrm{~min}$, and gradient-dehydrated in 70,85 and $100 \%$ ethanol.

The probe mix used for FISH analysis consisted of centromere enumeration probes (CEPs) of chromosomes 3, 7 and 17, and locus-specific identifier probes to the locus 9p21 of the tumor suppressor gene p16 (Beijing GP Medical Technologies, Ltd., Beijing, China). Under dry and dark conditions, the probe mixture [8 $\mu 1$ hybridization buffer solution (Hubei Taikang Medical Equipment Co., Ltd.) and $2 \mu \mathrm{l}$ probe] was prepared, and next denatured at $73^{\circ} \mathrm{C}$ for $5 \mathrm{~min}$ in an electric-heated thermostatic water bath (HH.S11-Ni2; Hubei Taikang Medical Equipment Co., Ltd.). The probe mixture was then added to the slides and incubated overnight $(>17 \mathrm{~h})$ at $42^{\circ} \mathrm{C}$ for hybridization. In order to prevent volatilization of the probe mixture volatilize, cover slips were used to cover the hybridized area, and sealing film was mounted in the surrounding gap.

The following day, the slides were dehydrated in $70 \%$ ethanol, and naturally dried prior to be stained with 4',6-diamidino-2-phenylindole (DAPI). Cells with three-color fluorescent hybridization signals (DAPI/fluorescein isothiocyanate/tetramethylrhodamine; fluorescence in situ hybridization detection kit; GP Medical Technologies, Ltd.) were observed under a microscope (BX51; Olympus Corporation, Tokyo, Japan). Image analysis was performed with VideoTesT-FISH 2.0 software (Digital Imaging Systems Ltd., Bourne End, UK).

All cases were screened by a cytotechnologist with specific training in FISH interpretation. Specimens were considered to be abnormal when presenting $>12$ cells with heterozygosity loss or homozygosis loss of solely p16, or $>4$ aneusomic cells of $>2$ other probes. Positive cases were confirmed by a second cytotechnologist. In addition, specimens were also considered to be abnormal if contained 25 cells with tetrasomy, which corresponded to 4 signals in each of the 4 probes analyzed.

Statistical analysis. Statistical analysis for evaluating the differences between cytology and FISH was performed using two-sided Fisher's exact test. When not applicable, $\chi^{2}$ test was performed instead (7). $\mathrm{P}<0.05$ was considered to indicate a statistically significant difference. All analyses were performed using SPSS version 13.0 (SPSS, Inc., Chicago, IL, USA).

\section{Results}

A total of 125 voided urine specimens from 125 patients were collected in the present study. The average follow-up time following cytology and FISH assay was 23.8 months (range, 5-39 months). Of the 125 patients, 8 could not be contacted until the last follow-up, and were therefore excluded from the study. The voided urine specimens from the remaining 117 patients were analyzed in the present study, 79 of whom were men and 38 women. Cytology could not be performed in 6 of the 117 samples due to insufficient number of cells in 
Table I. Cytology data compared with histology data $(n=117)$.

\begin{tabular}{lrrr}
\hline & \multicolumn{3}{c}{ Histology } \\
\cline { 2 - 4 } Cytology & Urothelial lesions & Negative & Total \\
\hline Positive & 8 & 0 & 8 \\
Negative & 10 & 93 & 103 \\
Nondiagnostic & 1 & 5 & 6 \\
Total & 19 & 98 & 117 \\
\hline
\end{tabular}

Table II. FISH data compared with histology data $(n=117)$.

\begin{tabular}{lrrr}
\hline & \multicolumn{3}{c}{ Histology } \\
\cline { 2 - 4 } FISH & Urothelial lesions & Negative & Total \\
\hline Positive & 16 & 4 & 20 \\
Negative & 2 & 88 & 90 \\
Nondiagnostic & 1 & 6 & 7 \\
Total & 19 & 98 & 117 \\
\hline
\end{tabular}

FISH, fluorescence in situ hybridization.

Table III. Sensitivity, specificity and predictive value of FISH data compared with cytology data.

\begin{tabular}{lccc}
\hline $\begin{array}{l}\text { Histology, } \% \\
(\mathrm{n}=117)\end{array}$ & Cytology, $\%$ & FISH, $\%$ & P-value \\
\hline Sensitivity & $42.11(8 / 19)$ & $84.21(16 / 19)$ & $0.01^{\mathrm{a}}$ \\
Specificity & $94.90(93 / 98)$ & $89.80(88 / 98)$ & 0.09 \\
False positive & $0.00(0 / 8)$ & $20.00(4 / 20)$ & 0.24 \\
False negative & $9.71(10 / 103)$ & $2.20(2 / 90)$ & $0.02^{\mathrm{a}}$ \\
PPV & $100.00(8 / 8)$ & $80.00(16 / 20)$ & 0.24 \\
NPV & $90.29(93 / 103)$ & $97.78(88 / 90)$ & $0.02^{\mathrm{a}}$
\end{tabular}

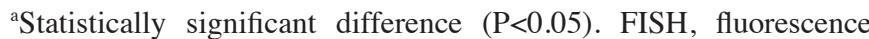
in situ hybridization; PPV, positive predictive value; NPV, negative predictive value.

these urine samples, while the fluorescence signals of FISH test in 7 specimens could not be interpreted. As an insufficient number of cells in urine is typically voided during FISH, all these patients, which were regarded as non-diagnostic, were included in the subsequent statistical analysis in order to estimate the overall sensitivity and specificity of FISH. Of the 117 patients, 19 had histologically confirmed UTUC, of whom, 6 exhibited stage pTa disease, 5 stage pT1 disease, 5 stage pT2 disease and 3 stage pTis disease (7 G1, 8 G2 and 4 G3).

Tables I and II indicate the characteristics of the study population. Table I contains the cytology data of 117 patients, while their FISH data is presented in Table II. Table III reveals the sensitivity, specificity and predictive value of FISH and cytology tests.
The overall sensitivity of FISH to detect UTUC from voided urine specimens was $84.21 \%$ (16/19), whereas that of cytology was $42.11 \%(8 / 19)(\mathrm{P}=0.01)$. The overall specificity of FISH to detect UTUC from voided urine specimens was $89.80 \%$ (88/98), compared with $94.90 \%$ (93/98) of cytology $(\mathrm{P}=0.09)$. Due to the limited number of specimens, the differences in grade and muscle-invasiveness of tumors were not compared. A total of 6 specimens could not be diagnosed by cytology ( 1 of which exhibited positive histology and 5 negative), as the cellular morphology was obscure or the number of cells in the sample was insufficient. A total of 7 specimens could not be diagnosed by FISH (1 of which exhibited positive histology and 6 negative), since their fluorescence signals were faint or the number of cells in the sample was insufficient.

In total, 10 false-negative samples were diagnosed by cytology. These samples were also analyzed by FISH, and 8 of them rendered a positive result. FISH analysis produced 2 false-negatives; these samples were also analyzed by cytology, and were negative. A total of 4 false-positive samples analyzed by FISH were also analyzed by cytology, and all rendered a negative result. The positive predictive value (PPV) and negative predictive value (NPV) of FISH were $80.00 \%$ $(16 / 20)(\mathrm{P}=0.24$ vs. cytology) and $97.78 \%(88 / 90)(\mathrm{P}=0.02$ vs. cytology), respectively, whereas the PPV and NPV of cytology were $100.00 \%$ (8/8) and 90.29\% (93/103), respectively.

Table IV presents the histopathological classification and other findings of 19 UTUCs in the last follow-up screening of 19 cases. Of these 19 patients (15 males and 4 females; mean age, 64 years; range, 48-82 years), 3 exhibited gross hematuria and 5 microhematuria. The average follow-up time was 21.42 months (range, 6-36 months). One patient experienced recurrence of bladder cancer in the left ureter after 33 months of bladder cancer excision.

Fig. 1 reveals abnormal and normal FISH signals of CEP3, CEP7, CEP17 and gene locus-specific p16. Fig. 1A and B represent tumor cells, while Fig. $1 \mathrm{C}$ and $\mathrm{D}$ represent normal cells.

\section{Discussion}

UTUC is not a frequent type of urological cancer, accounting for only $5 \%$ of all transitional cell carcinomas of the urinary system (12). UTUCs tend to present high grade and stage, and are usually associated with poor prognosis (12). Therefore, early diagnosis and effective treatment for UTUC is imperative $(4,13)$. ImmunoCyt/uCyt+ test, voided or clean catch urine cytology, radiographic or ultrasound imaging and ureteroscopy are the most common diagnostic methods for UTUC (7). However, urine cytology has been reported to be of little value in evaluating unclear or suspicious findings of the upper urinary tract obtained by imaging studies $(14,15)$, which have a sensitivity of $30-50 \%(16,17)$. Furthermore, radiographic and ultrasound imaging have low sensitivity for the detection of carcinoma in situ in small lesions, and ureteroscopy is an invasive method which patients prefer to avoid (4).

FISH was previously reported to have a high sensitivity and specificity for detecting bladder cancer (18). Few retrospective studies on FISH demonstrated that FISH had high sensitivity and specificity in detecting UTUC, contrarily to cytology $(4,7,10)$. However, the majority of those studies were 
Table IV. Histopathological classification and other findings of 19 upper tract urothelial carcinomas.

\begin{tabular}{|c|c|c|c|c|c|c|c|c|c|}
\hline No. & Gender & $\begin{array}{c}\text { Age } \\
\text { (years) }\end{array}$ & $\mathrm{H}$ & $\begin{array}{l}\text { Bladder } \\
\text { cancer } \\
\text { recurrence }\end{array}$ & $\begin{array}{c}\text { Location } \\
\text { of } \\
\text { lesion }\end{array}$ & $\begin{array}{l}\text { Follow-up } \\
\text { time } \\
\text { (months) }\end{array}$ & $\begin{array}{c}\text { TCC stage/ } \\
\text { grade }\end{array}$ & Cytology & FISH \\
\hline 1 & Male & 54 & $\mathrm{MH}$ & Yes & LU & 33 & $\mathrm{pT} 2 / \mathrm{G} 2$ & Positive & Positive \\
\hline 2 & Male & 68 & None & No & RRP & 28 & $\mathrm{pTa} / \mathrm{G} 1$ & Negative & Positive \\
\hline 3 & Male & 72 & None & No & LU & 36 & $\mathrm{pT} 2 / \mathrm{G} 3$ & Positive & Positive \\
\hline 4 & Female & 71 & None & No & LRP & 35 & $\mathrm{pT} 1 / \mathrm{G} 2$ & Negative & Positive \\
\hline 5 & Male & 65 & MH & No & LRP & 29 & $\mathrm{pTis} / \mathrm{G} 1$ & Positive & Negative \\
\hline 6 & Male & 82 & None & No & RRP & 26 & $\mathrm{pT} 1 / \mathrm{G} 2$ & Negative & Positive \\
\hline 7 & Male & 48 & $\mathrm{GH}$ & No & RRP & 31 & $\mathrm{pTis} / \mathrm{G} 1$ & Positive & Positive \\
\hline 8 & Female & 63 & None & No & LRP & 22 & $\mathrm{pTa} / \mathrm{G} 2$ & Negative & Positive \\
\hline 9 & Male & 77 & $\mathrm{GH}$ & No & LRP & 24 & $\mathrm{pT} 2 / \mathrm{G} 3$ & Positive & Positive \\
\hline 10 & Male & 65 & None & No & RU & 19 & $\mathrm{pT} 1 / \mathrm{G} 1$ & Positive & Negative \\
\hline 11 & Male & 51 & $\mathrm{MH}$ & No & RU & 24 & $\mathrm{pTa} / \mathrm{G} 1$ & Negative & Positive \\
\hline 12 & Female & 53 & None & No & LRP & 12 & $\mathrm{pT} 1 / \mathrm{G} 2$ & Negative & Positive \\
\hline 13 & Male & 73 & None & No & RU & 9 & $\mathrm{pTa} / \mathrm{G} 2$ & Negative & Positive \\
\hline 14 & Female & 61 & None & No & LRP & 8 & pTis/G1 & Negative & Positive \\
\hline 15 & Male & 56 & $\mathrm{MH}$ & No & RU & 16 & $\mathrm{pTa} / \mathrm{G} 2$ & Nondiagnostic & Nondiagnostic \\
\hline 16 & Male & 76 & None & No & LRP & 23 & $\mathrm{pT} 2 / \mathrm{G} 3$ & Negative & Positive \\
\hline 17 & Male & 49 & $\mathrm{GH}$ & No & RRP & 17 & $\mathrm{pTa} / \mathrm{G} 1$ & Negative & Positive \\
\hline 18 & Male & 68 & None & No & LRP & 9 & $\mathrm{pT} 1 / \mathrm{G} 3$ & Negative & Positive \\
\hline 19 & Male & 64 & $\mathrm{MH}$ & No & RRP & 6 & $\mathrm{pT} 2 / \mathrm{G} 2$ & Positive & Positive \\
\hline
\end{tabular}

$\mathrm{H}$, hematuria; TCC, transitional cell carcinoma; FISH, fluorescence in situ hybridization; GH, gross hematuria; MH, microhematuria; LU, left ureter; RU, right ureter; LRP, left renal pelvis; RRP, right renal pelvis.
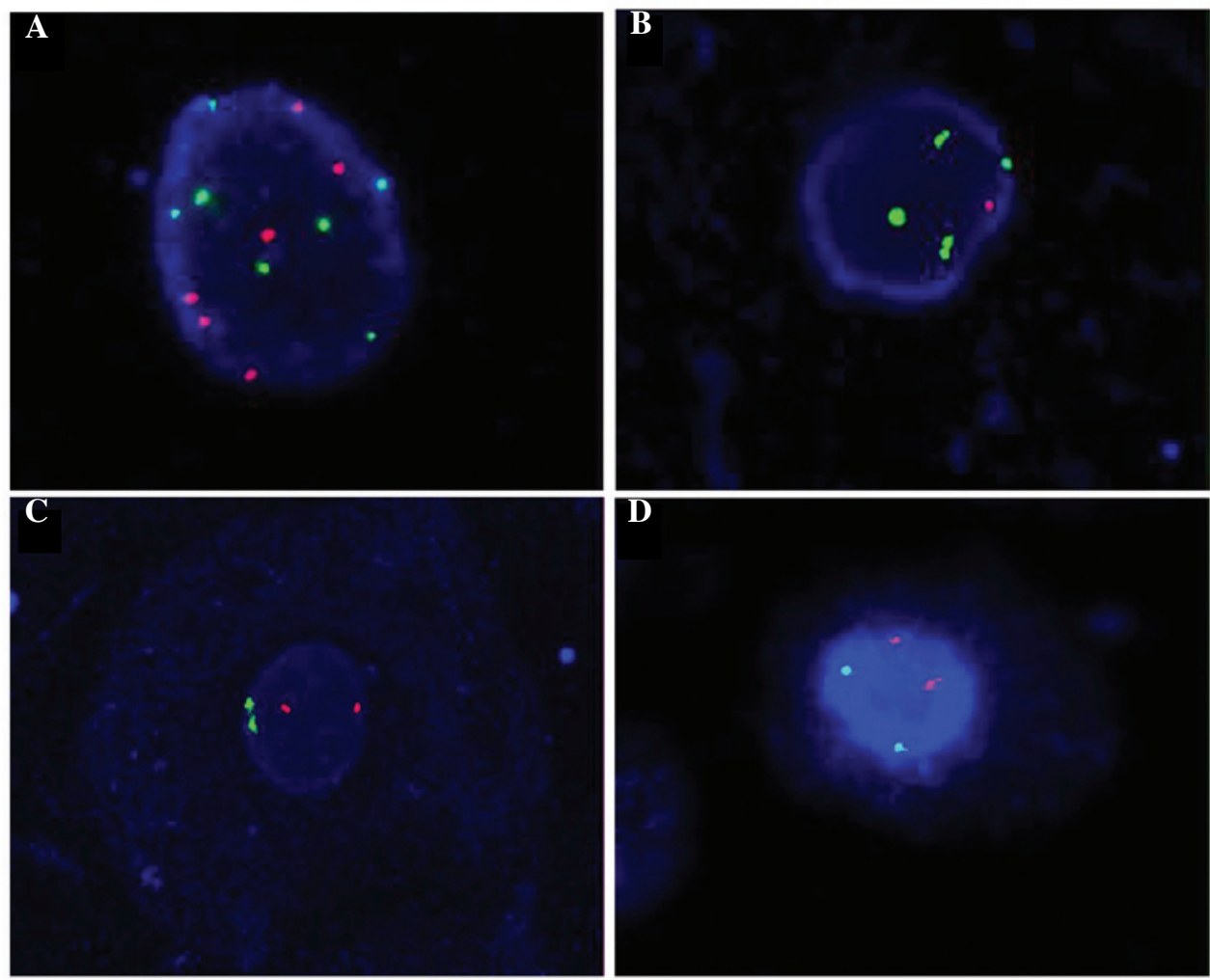

Figure 1. Representative images of abnormal and normal FISH signals of CEP3, CEP7, CEP17 and GLP p16. (A) Abnormal FISH signals of CEP3 (red, 6 dots) and CEP7 (green, 7 dots). (B) Abnormal FISH signals of GLP p16 (red, 1 dot) and CEP17 (green, 4 dots). (C) Normal FISH signals of CEP3 (red, 2 dots) and CEP7 (green, 2 dots). (D) Normal FISH signals of GLP p16 (red, 2 dots) and CEP17 (green, 2 dots). Magnification, x1,000. FISH, fluorescence in situ hybridization; CEP, centromere enumeration probe; GLP, gene locus-specific. 
retrospective, and the data available are insufficient $(4,7,10)$. Thus, prospective, randomized studies are required. In addition, specimens were detected mainly in clean catch urine, with a limited number of specimens being detected in voided urine (7). However, patients usually do not accept collecting clean catch urine, but would rather collect voided urine, particularly in China (7,19). In addition, reference standards of positive FISH are based on the diagnostic criteria of bladder cancer (9). However, exfoliative cells of the upper ureter or renal pelvis are present in low quantities in voided urine and are susceptible to contamination by bladder cells. Therefore, the diagnostic criteria of FISH in voided urine should be amended moderately.

In the present study, UTUC was detected in voided urine by FISH within a prospective randomized trial. If the number of cells following centrifugation was not sufficient for visual analysis with the naked eye, all the resuspended cells would be seeded onto glass slides, and the number of aneusomic cells in the whole slide would be counted, in order to increase FISH sensitivity. Combining microscopic observation with analysis of selected images enabled to exclude non-specific signals, thus improving FISH specificity. If numerous hemameba were present, samples would be observed using the DAPI channel with differential cell nucleus refractivity between epithelial cells and hemameba, in order to decrease the interference of hemameba.

In the present study, the sensitivity of FISH for detecting UTUC was higher than that of cytology $(\mathrm{P}=0.01)$, and the rate of false-negatives of FISH was lower than that of cytology $(\mathrm{P}=0.02)$. In addition, the NPV of FISH was superior to that of cytology $(\mathrm{P}=0.02)$. However, in terms of specificity, rate of false-positives and PPV, FISH was similar to cytology. Contrarily to a previous study by Huang et al (17), who reported a sensitivity of $100 \%$ for FISH in detecting UTUC, the sensitivity identified in the present study was low. This difference may be due to the fact that the majority of patients in the cohort analyzed by Huang et al (17) exhibited high-grade UTUCs, for which the FISH assay has higher sensitivity than for low-grade tumors $(17,20)$. The sensitivity of FISH for the detection of UTUC identified in the present study was similar to that reported by Gruschwitz et al (21), but higher than that reported by Reynolds et al (9). There is a possible reason for this difference. In the study by Reynolds et al (9), specimens were considered to be abnormal by FISH if 10 cells with near-tetrasomy or tetrasomy, or 5 cells with hypertetrasomy were detected. However, the cut-off value used in the present study was lower, based on the characteristics of the Chinese healthy donors who provided voided urine as a control. In the study by Reynolds et al (9), the samples consisted of ureteral washings and renal pelvis brushings, while voided urine specimens were used instead in the present study. Thus, the proportion of upper ureteral epitheliums as contaminated by bladder transitional epitheliums is low in the present study, compared with the study by Reynolds et al (9). Thus, the authors recommend in their study to apply a high cut-off value of $20 \%$, due to the enrichment in upper tract urothelial cells in their samples, whereas the cut-off value of $4 \%$ used in the present study still rendered high sensitivity in detecting patients with UUT-UCCs, due to the reduced number of false positives detected as a consequence of as a result of contamination by bladder transitional epitheliums in voided urine specimens. Therefore, the present authors recommend $4 \%$ as the cut-off value, which led to $84.21 \%$ sensitivity, $89.80 \%$ specificity, 2.20\% rate of false-negatives and $97.78 \%$ NPV for the detection of UTUCs in voided urine by FISH. However, this low cut-off value increased the false-positivity $(20.00 \%)$ and decreased the PPV (80.00\%) of FISH. In the present study, 2 false-negative results of FISH that were positive by cytology corresponded to low-grade tumor specimens, and 7 out of 10 false-negative results of cytology that were positive by FISH were high-grade tumor specimens. One specimen was nondiagnostic by FISH and cytology, due to an insufficient number of cells, but urethroscopy with biopsy during the follow-up demonstrated that it was a pTa G1 tumor in the right ureter.

In summary, the present data demonstrated that it is possible to apply FISH as a method to detect UTUC in voided urine specimens. FISH displayed good sensitivity and specificity when the cut-off value was $4 \%$, but this increased its rate of false-positivity and decreased its PPV. In the present study, FISH was observed to be a clinical reliable method for the detection of UTUC, with a higher sensitivity than cytology and equal specificity. In order to improve FISH specificity, non-specific signals were excluded between microscopic observation and analysis upon image selection. With regard to positive FISH results, further examination or follow-up are strongly recommended.

\section{Acknowledgements}

The present study was supported by grants from the National Natural Science Foundation of China (Beijing, China; grant no. 81100443), Chongqing Yuzhong District Science and Technology Plan (project no. 20120214) and Chongqing Municipal Health Bureau (scientific research project no. 2013-2-151).

\section{References}

1. Oya M and Kikuchi E; Committee for Establishment of Clinical Practice Guideline for Management of Upper Tract Urothelial Carcinoma; Japanese Urological Association: Evidenced-based clinical practice guideline for upper tract urothelial carcinoma (summary - Japanese Urological Association, 2014 edition). Int J Urol 22: 3-13, 2015.

2. Gupta R, Paner GP and Amin MB: Neoplasms of the upper urinary tract: A review with focus on urothelial carcinoma of the pelvicalyceal system and aspects related to its diagnosis and reporting. Adv Anat Pathol 15: 127-139, 2008.

3. Colin P, Koenig P, Ouzzane A, Berthon N, Villers A, Biserte J and Rouprêt M: Environmental factors involved in carcinogenesis of urothelial cell carcinomas of the upper urinary tract. BJU Int 104: 1436-1440, 2009.

4. Marín-Aguilera M, Mengual L, Ribal MJ, Musquera M, Ars E, Villavicencio H, Algaba F and Alcaraz A: Utility of fluorescence in situ hybridization as a non-invasive technique in the diagnosis of upper urinary tract urothelial carcinoma. Eur Urol 51: 409-415, 2007.

5. Mills IW, Laniado ME and Patel A: The role of endoscopy in the management of patients with upper urinary tract transitional cell carcinoma. BJU Int 87: 150-162, 2001

6. Wiener HG, Mian C, Haitel A, Pycha A, Schatzl G and Marberger M: Can urine bound diagnostic tests replace cystoscopy in the management of bladder cancer? J Urol 159: 1876-1880, 1998.

7. Mian C, Mazzoleni G, Vikoler S, Martini T, Knüchel-Clark R, Zaak D, Lazica A, Roth S, Mian M and Pycha A: Fluorescence in situ hybridisation in the diagnosis of upper urinary tract tumours. Eur Urol 58: 288-292, 2010. 
8. Sobin LH and Wittekind $\mathrm{CH}$ (eds): Urological tumours: Bladder In: TNM Classification of Malignant Tumours. 6th edition. John Wiley \& Sons, Hoboken, NJ, pp199-202, 2002.

9. Reynolds JP, Voss JS, Kipp BR, Karnes RJ, Nassar A, Clayton AC Henry MR, Sebo TJ, Zhang J and Halling KC: Comparison of urine cytology and fluorescence in situ hybridization in upper urothelial tract samples. Cancer Cytopathol 122: 459-467, 2014.

10. Luo B, Li W, Deng CH, Zheng FF, Sun XZ, Wang DH and Dai YP: Utility of fluorescence in situ hybridization in the diagnosis of upper urinary tract urothelial carcinoma. Cancer Genet Cytogenet 189: 93-97, 2009.

11. May M, Hakenberg OW, Gunia S, Pohling P, Helke C, Lübbe L, Nowack R, Siegsmund M and Hoschke B: Comparative diagnostic value of urine cytology, UBC-ELISA, and fluorescence in situ hybridization for detection of transitional cell carcinoma of urinary bladder in routine clinical practice. Urology 70: 449-453, 2007.

12. Oosterlinck W, Solona E, van der Meijden AP, Sylvester R, Böhle A Rintala E and Lobel B; European Association of Urology: EAU guidelines on diagnosis and treatment of upper tract transitional cell carcinnoma. Eur Urol 46: 147-154, 2004.

13. Stewart GD, Bariol SV, Grigor KM, Tolley DA and McNeill SA: A comparison of the pathology of transitional cell carcinoma of the bladder and upper urinary tract. BJU Int 95: 791-793, 2005.

14. Akkad T, Brunner A, Pallwein L, Gozzi C, Bartsch G, Mikuz G, Steiner $\mathrm{H}$ and Verdorfer I: Fluorescence in situ hybridization for detecting upper urinary tract tumors - a preliminary report. Urology 70: 753-757, 2007.
15. Zincke H, Aguilo JJ, Farrow GM, Utz DC and Khan AU: Significance of urinary cytology in the early detection of transitional cell cancer of the upper urinary tract. J Urol 116: 781-783, 1976

16. Lodde M, Mian C, Wiener H, Haitel A, Pycha A and Marberger M: Detection of upper urinary tract transitional cell carcinoma with ImmunoCyt: A preliminary report. Urology 58: 362-366, 2001.

17. Huang WT, Li LY, Pang J, Ruan XX, Sun QP, Yang WJ and Gao X: Fluorescence in situ hybridization assay detects upper urinary tract transitional cell carcinoma in patients with asymptomatic hematuria and negative urine cytology. Neoplasma 59: 355-360, 2012.

18. Song M-J, Lee H-M and Kim S-H: Clinical usefulness of fluorescence in situ hybridization for diagnosis and surveillance of bladder cancer. Cancer Genet Cytogenet 198: 144-150, 2010.

19. Shan Z, Wu P, Zheng S, Tan W, Zhou H, Zu Y, Qi H, Zhang P, Peng $\mathrm{H}$ and Wang Y: Evaluation of upper urinary tract tumors by FISH in Chinese patients. Cancer Genet Cytogenet 203: 238-246, 2010

20. Chen AA and Grasso M: Is there a role for FISH in the management and surveillance of patients with upper tract transitional-cell carcinoma? J Endourol 22: 1371-1374, 2008.

21. Gruschwitz T, Gajda M, Enkelmann A, Grimm MO, Wunderlich H, Horstmann M and Junker K: FISH analysis of washing urine from the upper urinary tract for the detection of urothelial cancers. Int Urol Nephrol 46: 1769-1774, 2014. 